\title{
ANÁLISIS DE LA CALIDAD DEL AGUA EN RÍOS DE LA CUENCA CHANCAY - LAMBAYEQUE, PERÚ
}

ANALYSIS OF WATER QUALITY IN RIVERS OF THE CHANCAY - LAMBAYEQUE BASIN, PERU

Ruben Gilberto Rodríguez Flores 1

(iD) https://orcid.org/0000-0002-4799-0203

ruben.rodriguez@unmsm.edu.pe.

\begin{abstract}
RESUMEN
La evaluación de la calidad del agua en la cuenca Chancay - Lambayeque, ubicada en la zona norte del Perú, es realizada por medio de los valores de diversos parámetros fisicoquímicos y microbiológicos con relación a los Estándares Nacionales de Calidad Ambiental para agua (ECA-agua), el objetivo del estudio fue analizar y evaluar la calidad ambiental de los cuerpos de agua de dicha cuenca, no solo en términos de sus principales parámetros fisicoquímicos y microbiológicos sino a través de la aplicación de índices de calidad del agua, para lo cual se utilizó tanto el índice NFS-WQI (National Sanitation Foundation - Water Quality Index) como el índice ICA-PE (Índice de Calidad del Agua de Perú). En dicha cuenca, los parámetros de los principales ríos se encuentran por debajo de los valores ECAs-agua (respecto a la Categoría 3: para riego de vegetales y bebida de animales), en lo que respecta a las quebradas, estas presentan un $\mathrm{pH}$ menor a 6,5 asícomo parámetros con valores superiores al ECA-agua para cuatro elementos metálicos como son el Aluminio, Hierro, Cobre y Zinc. Por su parte cuando se determinaron los dos índices de calidad (NSF-WQI e ICA-PE) para los ríos y quebradas de la cuenca, estos alcanzaron la calificación de buena..
\end{abstract}

Keywords: Cuenca Chancay-Lambayeque, índice de calidad, ICA-PE, NSF-WQI..

\begin{abstract}
The evaluation of water quality in the Chancay - Lambayeque basin, located in the northern part of Peru, is carried out by means of the values of various physicochemical and microbiological parameters in relation to the National Environmental Quality Standards for water (EQS-water), the objective of the study was to analyse and evaluate the environmental quality of the water bodies in this
\end{abstract}

\footnotetext{
${ }^{1}$ Facultad de Ingeniería Ambiental y de Recursos Naturales. Universidad Nacional del Callao. Av. Juan Pablo II 306 Bellavista.Callao - Perú. E-mail: rgrodriguezf@unac.edu.pe Unidad de Posgrado de la Facultad de Ingeniería Geológica Minera, Metalúrgica y Geográfica. Universidad Nacional Mayor deSan Marcos. Av. Venezuela s/n cuadra 24, Lima - Perú.
} 
basin, not only in terms of their main physicochemical and microbiological parameters, but also through the application of water quality indices, for which both the NFS-WQI (National Sanitation Foundation - Water Quality Index) and the ICA-PE index (Peruvian Water Quality Index) were used. In this basin, the parameters of the main rivers are bel ow the ECA-water values (with respect to Category 3: for irrigation of vegetables and drinking water for animals), with respect to the streams, these have a pH of less than 6.5 and parameters with values higher than the EQS-water for four metallic elements such as Aluminium, Iron, Copper and Zinc. On the other hand, when the two quality indices (NSF-WQI and ICA-PE) were determined for the rivers and streams of the basin, these reached the qualification of good.

Keywords: Chancay-Lambayeque basin, quality index, ICA-PE index, NSF-WOI index.

\section{INTRODUCCIÓN}

En la gestión de los recursos hídricos es importante conocer la condición ambiental de un cuerpo de agua, para ello el uso de un índice de calidad se convierte en una herramienta útil para el manejo y la toma de decisiones sobre la calidad y uso potencial que se les puede dar a los cuerpos de agua, aun cuando tengan diversos niveles de contaminación (Abassi, 2012), dicho índice debe recoger de manera simple y representativa la condición de diversos parámetros fisicoquímicos y microbiológicos (Sargaonkar and V. Deshpande, 2003, ŠtambukGiljanović N., 2003).

Los Índices de Calidad del Agua (WQI) más importantes utilizados en todo el mundo son el método WQI aritmético ponderado (WAWQI) (Brown et al., 1970), el Índice del Operador Mínimo (MOI) (Smith 1990), el WQI de la National Sanitation Foundation (NSF-WQI), el WQI del Consejo Canadiense de Ministros del Ambiente (CCME-WQI) (Canadian Council of Minister of the Environment, 2001) y el WQI de Oregon (OWQI) (Cude, 2001).

EI WQI (Water Quality Index) es una herramienta aritmética usada para transformar una cantidad de datos de calidad en un simple número, y que representa un cierto nivel de calidad del agua (Bordalo, 2006), eliminando las evaluaciones subjetivas de dicha calidad (Štambuk-Giljanović N., 2003).

El concepto de WQI fue desarrollado y propuesto por primera vez por Horton (1965), y es un numero único que expresa la calidad general del agua en un área y tiempo determinado en función de varios parámetros de calidad del agua, el mismo que permite transmitir información al público en general (Štambuk-Giljanović N., 2003). Cuando se consideran sus características y limitaciones especificas (Ott 1978, Hallock, 2002, Pesce \& Wunderlin, 2000). En general todos ellos consideran similares parámetros, pero difieren en su tratamiento, integración e interpretación (Pandey \& Sundaram 2002, Ram \& Anandh 1996).

Los índices de calidad del agua son calculados en dos etapas, en la primera, hay una transformación de los parámetros que caracterizan el agua, para después y aplicando los valores de subíndices hacer la determinación del valor correspondiente al índice de calidad del agua (Brown et al., 1972, Bordalo et al., 2001, Cude, 2001, Liou et al., 2004, El-Gafy et al., 2005, Sinha \& Sanexa, 2006, Gomez et al., 2007, Fulazzaky, 2009, Rodriguez, 2019). 


\section{METOdOLOGÍA}

La presente investigación ha tomado en cuenta los resultados de los monitoreos participativos de los años 2013, 2014, 2015 y 2016 en ríos y quebradas de la cuenca Chancay - Lambayeque a cargo de la Autoridad Administrativa del Agua (AAA) perteneciente a la Autoridad Nacional del Agua (ANA) en el marco del Proyecto de Modernización de la Gestión de los Recursos Hídricos, las mismas que se han realizado des de la naciente de la cuenca hasta la desembocadura en el Océano Pacifico. Los monitoreos se han realizado en 19 puntos, de los cuales 15 puntos corresponden a ríos y cuatro puntos restantes a quebradas, los cuales están descritos en la tabla 1.

\section{Tabla 1}

Puntos de monitoreo en ríos y quebradas de la cuenca Chancay - Lambayeque

\begin{tabular}{|c|c|c|c|c|}
\hline \multirow{2}{*}{$\begin{array}{c}\text { Rioo } \\
\text { Quebrada } \\
\end{array}$} & \multirow{2}{*}{ Descripción } & \multicolumn{2}{|c|}{ Coordenadas UTM } & \multirow{2}{*}{$\begin{array}{c}\text { Altitud } \\
\text { (m.s.n.m) }\end{array}$} \\
\hline & & Norte & Este & \\
\hline & Rio Chancay, naciente del rio aguas debajo & & & \\
\hline $\mathrm{R} 1$ & de la Laguna Morococha & 757670 & 9247794 & 3708 \\
\hline $\mathrm{R} 2$ & Rio Cedro, altura Mina La Zanja & 731397 & 9244733 & 3240 \\
\hline R3 & RioSan Juan Pampa, antes de rio Chancay & 745123 & 9265047 & 2010 \\
\hline R4 & Rio Conramar, altura distrito Catilluc & 744856 & 9246814 & 2793 \\
\hline R5 & $\begin{array}{l}\text { Rio Chancay, altura Puente Lucma } \\
\text { Rio Chorro Blanco, después de canal del }\end{array}$ & 741651 & 9253958 & 2479 \\
\hline R6 & rio Pisit & 735583 & 9256347 & 2583 \\
\hline R7 & Rio Las Nieves, altura distrito la Esperanza & 731353 & 9271881 & 1588 \\
\hline $\mathrm{R} 8$ & Rio Cañad, $100 m$ de puente Cañad & 721416 & 9264567 & 1185 \\
\hline R9 & Rio San Lorenzo, $50 \mathrm{~m}$ de puente Barandas & 714387 & 9262886 & 977 \\
\hline R10 & Rio Chancay, antes de rio San Lorenzo & 714412 & 9262886 & 978 \\
\hline $\mathrm{R} 11$ & Rio Cumbil & 693915 & 9270408 & 395 \\
\hline $\mathrm{R} 12$ & Rio Chancay, puente San Carlos & 691110 & 9268397 & 334 \\
\hline R13 & Rio Chancay, La Puntilla & 665843 & 9254348 & 172 \\
\hline & Rio Lambayeque, $200 \mathrm{~m}$ antes de Toma & & & \\
\hline R14 & Santeño & 645376 & 9251766 & 65 \\
\hline R15 & $\begin{array}{l}\text { Rio Reque, después de canal Eten } \\
\text { Quebrada La Pampa, aguas arriba de }\end{array}$ & 629154 & 9241146 & 20 \\
\hline Q1 & $\begin{array}{l}\text { vertimiento autorizado de minera } \\
\text { Quebrada La Pampa, aguas abajo de }\end{array}$ & 732752 & 9245304 & 3264 \\
\hline Q2 & vertimiento autorizado minera & 732478 & 9245713 & 3263 \\
\hline Q3 & $\begin{array}{l}\text { Quebrada Colorada, } 500 \text { m después de } \\
\text { pasivos a mbientales } \\
\text { Quebrada Colorada, altura de puente } \\
\text { carretera a Chota, aguas abajo de pasivos }\end{array}$ & 758048 & 9257031 & 3776 \\
\hline Q4 & ambientales de Sinchao & 756307 & 9258607 & 3576 \\
\hline
\end{tabular}

Fuente: Adaptado de Informes Técnicos participativos de la calidad del agua superficial en la cuenca Chancay Lambayeque (ANA, 2013, ANA, 2014, ANA, 2015, ANA, 2016)

El monitoreo de la calidad del agua de ríos y quebradas se ha realizado según el Protocolo Nacional de Monitoreo de la Calidad de los Cuerpos Naturales de Agua Superficial aprobado por Resolución (MINAGRI, 2016). La localización de los ríos y quebradas se puede apreciar en la figura 1. 
Figura 1

Localización de los puntos de monitoreo en ríos y quebradas de la cuenca Chancay - Lambayeque ubicada en la zona norte del Perú.

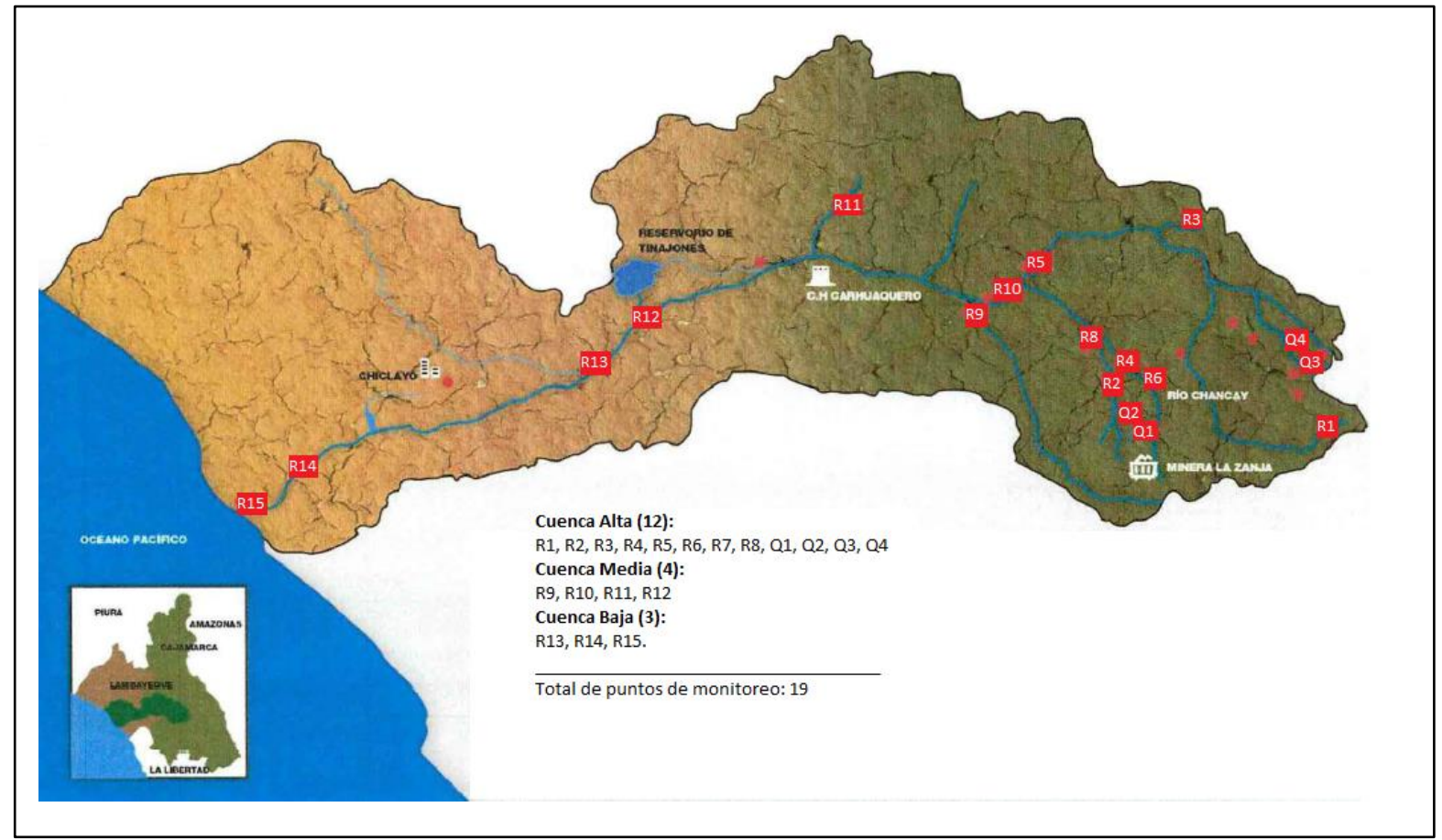

Para el análisis de las características fisicoquímicas y microbiológicas de los ríos y quebradas de la cuenca se han tomado en cuenta los ECAs en lo que corresponde a la Categoría 3 sobre riego de vegetales y bebida de animales, aprobado por el Decreto Supremo $\mathrm{N}^{\circ}$ 004-2017-MINAM (MINAM, 2017)

La calidad ambiental del agua expresado en términos índices de calidad fue determinada según sus respectivas metodologías, en lo que respecta al índice ICA-PE, se utilizó la metodología para la determinación del índice de calidad de agua ICA-PE, aplicado a los cuerpos de agua continentales superficiales (ANA, 2018) y para el índice NSF-WQI la metodología aprobada por la National Sanitation Foundation de los Estados Unidos (Călmuc et al, 2018).

\section{Cálculo del Índice de Calidad de Agua en Perú (ICA-PE)}

El índice ICA-PE fue establecido en el 2018 por la Autoridad Nacional del Agua (ANA) y está basado en la formula canadiense emitida por el Canadian Council of Ministers of the Environment (CCME) y modificada por los Ministerios del Ambiente de Alberta y Columbia Británica (provincia de Canadá) (ANA, 2018).

En la determinación del índice ICA-PE se toma en cuenta tres factores (alcance, frecuencia y amplitud), lo que resulta del cálculo matemático un valor único (entre 0 y 100), que va a representar y describir el estado de la calidad del agua de un punto de monitoreo, un curso de agua, un rio o cuenca (ANA, 2018). 


$$
I C A-P E=100-\left(\sqrt[2]{\frac{F_{1}^{2}+F_{2}^{2}+F_{3}^{2}}{3}}\right)
$$

Donde:

ICA-PE es el valor del índice, $F_{1}$ es el alcance o cantidad de parámetros que no cumplen los Estándares de Calidad Ambiental (ECA) del agua, F2 es la frecuencia o cantidad de datos que no cumplen el ECA y $F_{3}$ la amplitud o exceso de todos los datos respecto al número total de datos.

\section{Cálculo del Índice de Calidad del agua NSF-WQI}

Es uno de los índices que se utilizan para determinar la calidad del agua es el NSF-WQI (National Sanitation Foundation - Water Quality Index), este índice tiene la escala de 0 a 100 y se considera un índice decreciente porque sus valores se reducen al aumentar la contaminación de agua. El índice incluye la valoración de nueve (09) parámetros, como son $\mathrm{DBO}, \mathrm{OD}, \mathrm{NO}_{3}, \mathrm{PO}_{4}, \mathrm{~T}$, Tur., TS, $\mathrm{pH}$ y CF, fue desarrollado por Brown et al. (1972).

La siguiente ecuación es usada para obtener el valor del NSF-WQI:

$$
\mathrm{NFS}-\mathrm{WQI}=\mathrm{II}_{\mathrm{i}=1}^{\mathrm{n}} \mathrm{WQI}_{\mathrm{i}}^{\mathrm{w}_{\mathrm{i}}}
$$

Donde:

NSF-WQI es el valor del índice, WQI i es el valor del índice relacionado para el parámetro de calidad $\mathrm{i}, \mathrm{w}_{\mathrm{i}}$ es el coeficiente de ponderación relacionado con parámetro de calidad i, y $\mathrm{n}$ es el número de WQP (Water Quality Parameter).

\section{RESULTADOS Y DISCUSIÓN}

\section{Características fisicoquímicas y microbiológicas de los cuerpos de agua}

Los ríos de la cuenca muestran un pH mayor que el de quebradas, pero dentro del rango del ECA, el oxígeno disuelto (OD), conductividad (Cond), Demanda Biológica de Oxigeno (DBO) y Demanda Química de Oxigeno (DQO) no han sobrepasado el ECA respectivo. En cuanto a los Coliformes termotolerantes (CF), estos no han sobrepasado el ECA con excepción del valor promedio alcanzado por los ríos en el año 2015. En lo que corresponde a los 17 metales monitoreados tanto en ríos como en quebradas estos no han sobrepas ado el ECA respectivo, con excepción de los valores determinados para cuatro (4) metales como son Aluminio (Al), Hierro (Fe), Cobre ( $\mathrm{Cu}$ ) y Zinc ( $\mathrm{Zn}$ ) en las quebradas para los años de estudio, esta variación se muestra en la tabla 2. 
Tabla 2

Promedio de características fisicoquímicas y microbiológicas en ríos y quebradas (Queb) de la cuenca Chancay - Lambayeque durante los años 2013, 2014, 2015 y 2016

\begin{tabular}{|c|c|c|c|c|c|c|c|c|c|c|}
\hline \multirow{2}{*}{ Parámetro } & \multirow{2}{*}{ Unidad } & \multirow{2}{*}{$\begin{array}{c}\text { ECA } \\
\text { (categoría } \\
3 \text { ) } \\
\end{array}$} & \multicolumn{2}{|c|}{2013} & \multicolumn{2}{|c|}{2014} & \multicolumn{2}{|c|}{2015} & \multicolumn{2}{|c|}{2016} \\
\hline & & & Rio & Queb. & Rio & Queb. & Rio & Queb. & Rio & Queb. \\
\hline $\mathrm{pH}$ & & $6.5-8.5$ & 7.4 & 5.3 & 8.5 & 4.8 & 8.3 & 5.4 & 8.3 & 4.6 \\
\hline OD & $\mathrm{mg} / \mathrm{l}$ & $\geq 4$ & 7.8 & 7.2 & 8.1 & 7.1 & 8.1 & 7.1 & 6.8 & 4.3 \\
\hline Cond. & $\mu \mathrm{S} / \mathrm{cm}$ & 2500 & 702.7 & 200.9 & 257.9 & 561.1 & 257.9 & 561.1 & 287.0 & 240.9 \\
\hline $\mathrm{CF}$ & $\mathrm{NMP} / 100 \mathrm{ml}$ & 1000 & 946 & 2 & 122 & 2 & 8543 & 2 & 179 & 2 \\
\hline DBO & $\mathrm{mg} / \mathrm{L}$ & 15 & 2 & 2 & 4 & 2 & 4 & 2 & 3 & 3 \\
\hline DQO & $\mathrm{mg} / \mathrm{L}$ & 40 & 9.8 & 7.8 & 12.2 & 27.1 & 12.2 & 21.7 & 6.0 & 6.0 \\
\hline Al & $\mathrm{mg} / \mathrm{L}$ & 5 & 0.72 & 1.43 & 0.11 & 5.10 & 0.12 & 8.93 & 0.16 & 4.66 \\
\hline As & $\mathrm{mg} / \mathrm{L}$ & 0.1 & 0.003 & 0.005 & 0.002 & 0.006 & 0.007 & 0.051 & 0.007 & 0.012 \\
\hline $\mathrm{Ba}$ & $\mathrm{mg} / \mathrm{L}$ & 0.7 & 0.038 & 0.026 & 0.026 & 0.033 & 0.026 & 0.033 & 0.031 & 0.027 \\
\hline $\mathrm{Be}$ & $\mathrm{mg} / \mathrm{L}$ & 0.1 & 0.001 & 0.001 & 0.001 & 0.001 & 0.001 & 0.001 & 0.001 & 0.001 \\
\hline B & $\mathrm{mg} / \mathrm{L}$ & 1 & 0.114 & 0.002 & 0.091 & 0.006 & 0.091 & 0.006 & 0.136 & 0.025 \\
\hline $\mathrm{Cd}$ & $\mathrm{mg} / \mathrm{L}$ & 0.01 & 0.000 & 0.003 & 0.000 & 0.009 & 0.000 & 0.028 & 0.000 & 0.004 \\
\hline Co & $\mathrm{mg} / \mathrm{L}$ & 0.05 & 0.001 & 0.004 & 0.000 & 0.018 & 0.000 & 0.025 & 0.001 & 0.025 \\
\hline $\mathrm{Cu}$ & $\mathrm{mg} / \mathrm{L}$ & 0.2 & 0.007 & 0.705 & 0.007 & 1.489 & 0.008 & 5.240 & 0.017 & 0.694 \\
\hline $\mathrm{Cr}$ & $\mathrm{mg} / \mathrm{L}$ & 0.1 & 0.001 & 0.001 & 0.000 & 0.001 & 0.000 & 0.001 & 0.001 & 0.004 \\
\hline $\mathrm{Fe}$ & $\mathrm{mg} / \mathrm{L}$ & 5 & 0.5 & 2.4 & 0.1 & 16.6 & 0.2 & 22.5 & 0.1 & 16.4 \\
\hline $\mathrm{Li}$ & $\mathrm{mg} / \mathrm{L}$ & 2.5 & 0.006 & 0.002 & 0.012 & 0.004 & 0.012 & 0.004 & 0.014 & 0.004 \\
\hline $\mathrm{Mn}$ & $\mathrm{mg} / \mathrm{L}$ & 0.2 & 0.036 & 0.590 & 0.024 & 4.604 & 0.029 & 4.798 & 0.048 & 7.108 \\
\hline $\mathrm{Hg}$ & $\mathrm{mg} / \mathrm{L}$ & 0.001 & 0.000 & 0.000 & 0.000 & 0.000 & 0.000 & 0.000 & 0.000 & 0.000 \\
\hline $\mathrm{Ni}$ & $\mathrm{mg} / \mathrm{L}$ & 0.2 & 0.001 & 0.003 & 0.000 & 0.005 & 0.000 & 0.005 & 0.002 & 0.016 \\
\hline $\mathrm{Pb}$ & $\mathrm{mg} / \mathrm{L}$ & 0.05 & 0.001 & 0.004 & 0.000 & 0.017 & 0.000 & 0.017 & 0.001 & 0.013 \\
\hline $\mathrm{Se}$ & $\mathrm{mg} / \mathrm{L}$ & 0.02 & 0.000 & 0.000 & 0.000 & 0.000 & 0.000 & 0.000 & 0.001 & 0.002 \\
\hline $\mathrm{Zn}$ & $\mathrm{mg} / \mathrm{L}$ & 2 & 0.017 & 0.506 & 0.017 & 1.291 & 0.021 & 4.683 & 0.035 & 6.619 \\
\hline
\end{tabular}

Comportamiento del $\mathrm{pH}$

En cuanto al pH, en los ríos de la cuenca Chancay-Lambayeque y durante los años de estudio el valor se ha mantenido dentro de los rangos del ECA para la Categoría 3 (Riego de vegetales y bebida de animales), por el contrario, en las quebradas el pH, en el mismo periodo, ha presentado una mayor variación, principalmente por debajo del valor de 6,5 (pH mínimo) hasta alcanzar un valor de 2,9 de pH en el año 2014, evidenciando que se trata de corrientes de agua con un nivel de acidez considerable (ver figura 2 ).

Figura 2

Distribución del pH en ríos y quebradas de la cuenca Chancay - Lambayeque
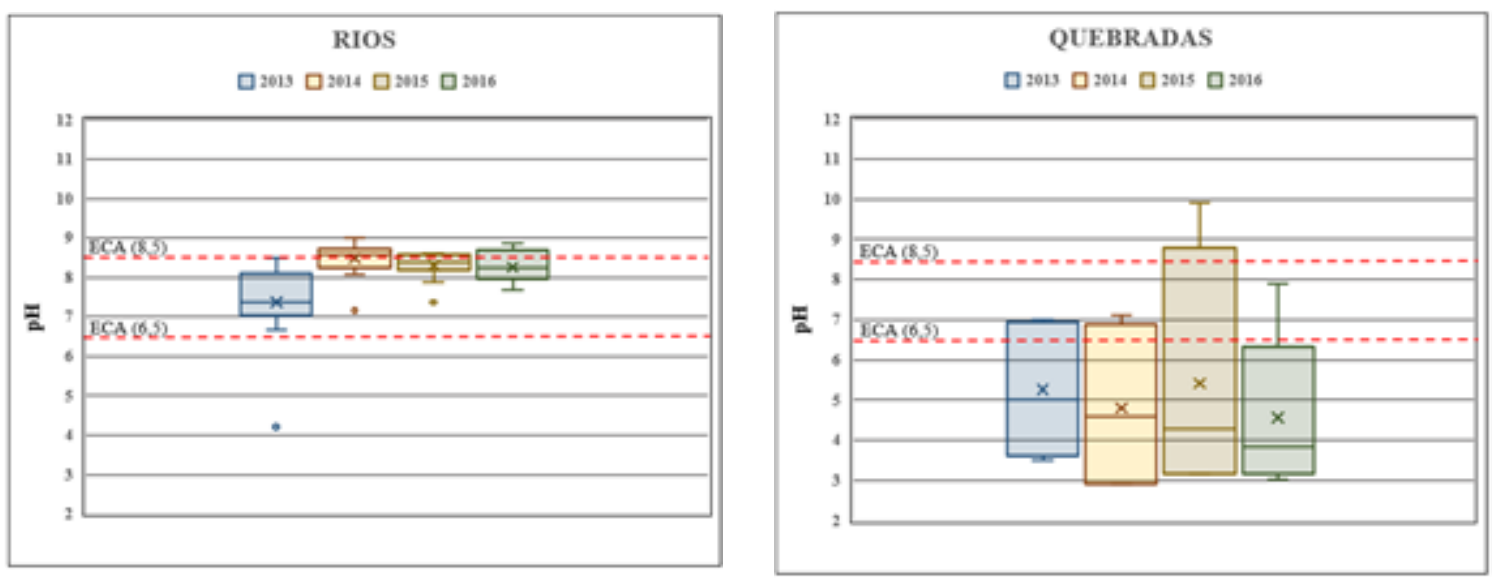
Comportamiento de metales

Respecto a la presencia de algunos metales en ríos y quebradas, estos se han manifestado de distinta manera, las concentraciones registradas en los ríos se encuentran por debajo del valor ECA, no siendo así para el caso de quebradas, donde se han presentado valores superiores al ECA tanto para el $\mathrm{AL}, \mathrm{Fe}, \mathrm{Cu}$ y $\mathrm{Zn}$, este comportamiento durante los cuatro años se puede observar en las figuras $3,4,5$ y 6 respectivamente.

Figura 3

Distribución del Aluminio (Al) en ríos y quebradas de la cuenca Chancay - Lambayeque
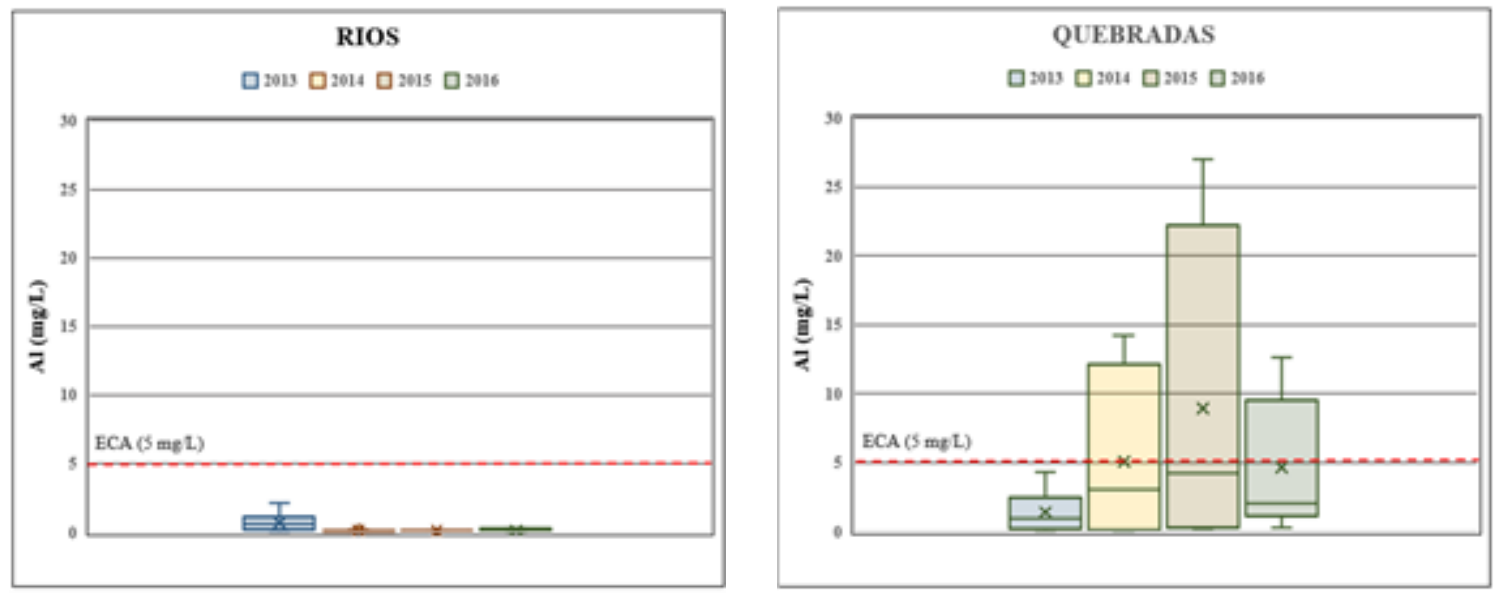

Figura 4

Distribución del Hierro (Fe) en ríos y quebradas de la cuenca Chancay - Lambayeque
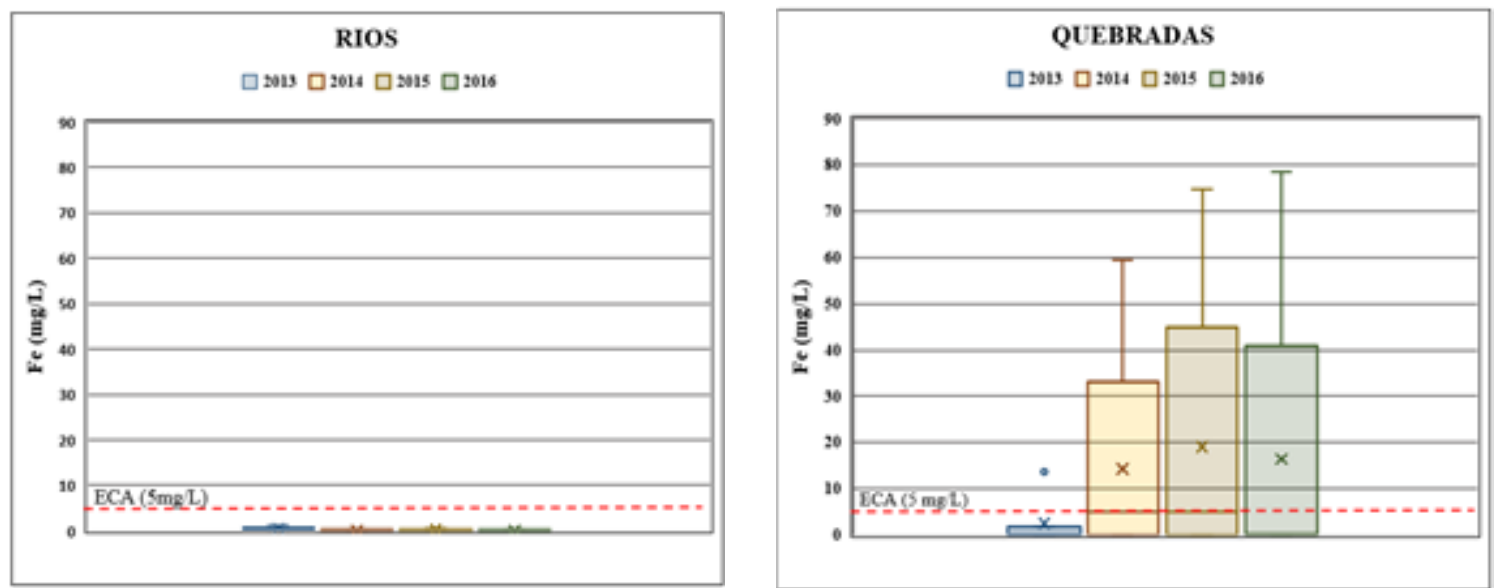

Las mayores concentraciones de Al, Fe y Cu se han dado en el año 2015, mientras que para el Zn ocurrió en el año 2016, esta elevada concentración de metales podría deberse a la existencia de Pasivos Ambientales en están presentes en las quebradas de la parte alta de la cuenca tal como lo indican los informes de Monitoreo participativo (ANA, 2013, ANA, 2014, ANA, 2015, ANA, 2016), que en periodo de lluvia provocan el ingreso de contaminantes a los cuerpos de agua en la parte alta de la cuenca. 
Figura 5

Distribución del Cobre $(\mathrm{Cu})$ en ríos y quebradas de la cuenca Chancay - Lambayeque
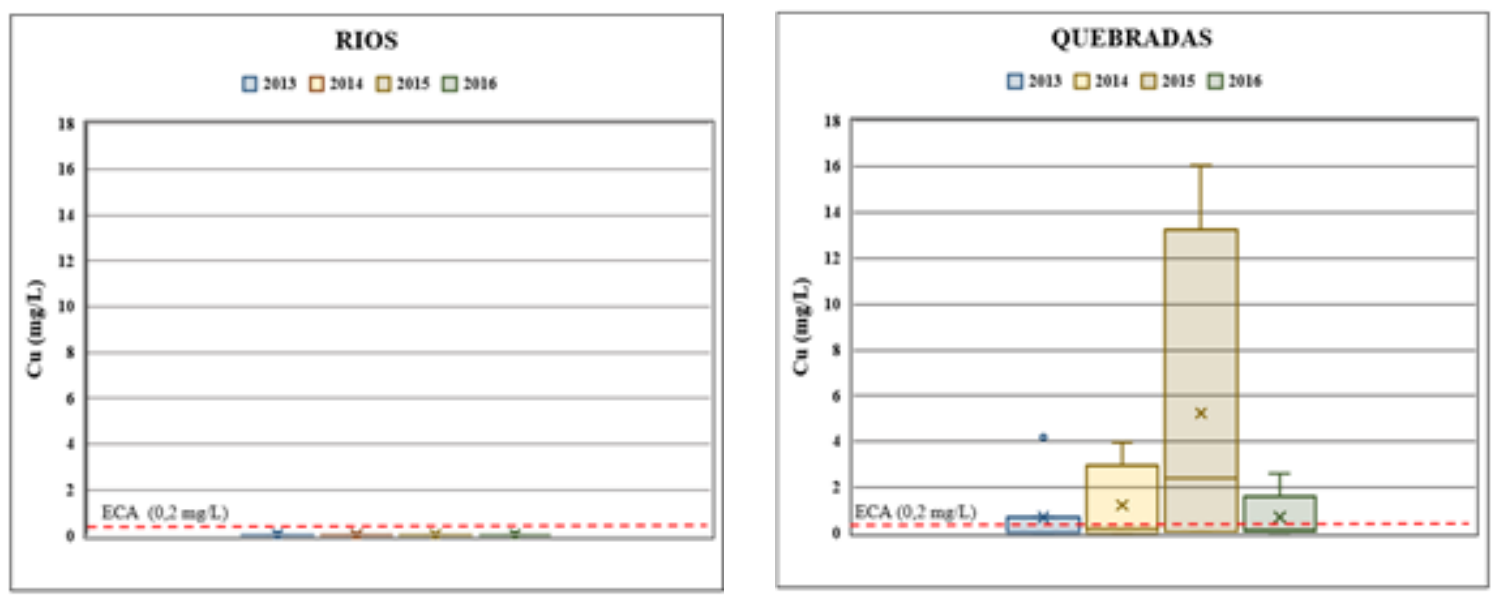

Figura 6

Distribución del Zinc (Zn) en ríos y quebradas de la cuenca Chancay - Lambayeque
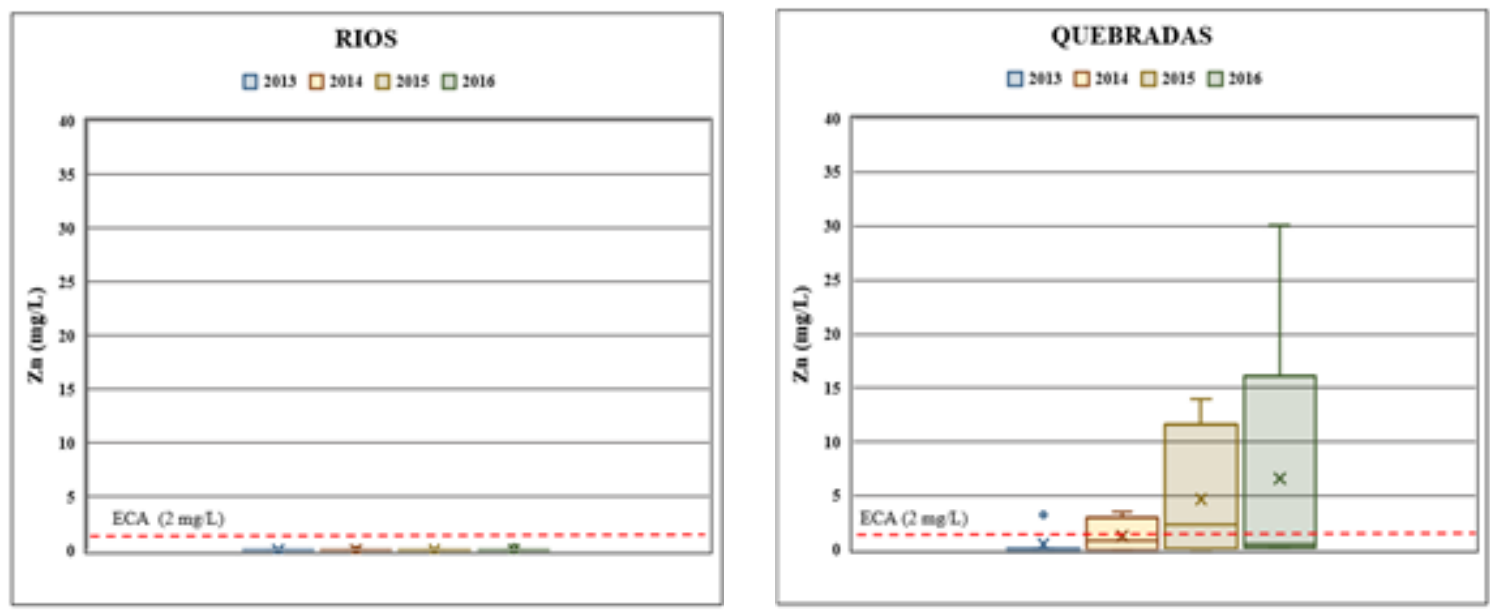

En la cuenca Chancay - Lambayeque, la mayoría de parámetros en los ríos cumplen con no pasar los valores ECA de la Categoría 3 (Riego de vegetales y bebida de animales), sin embargo, para el caso de las quebradas tanto el pH como de 4 elementos ( $\mathrm{Al}, \mathrm{Fe}, \mathrm{Cu}$ y $\mathrm{Zn}$ ) no cumplen los respectivos valores ECA por la presencia de pasivos ambientales de antiguas actividades mineras (Quebrada Colorada) y con vertidos autorizados de aguas residuales tratadas (Quebrada La Pampa). Estos resultados son similares a los obtenidos por Olguin et al. (2010) para el rio Sordo en Xalapa (Veracruz- México) que para el caso del pH se mantuvo en un rango estable de 6,03 a 6,86 en época seca y de 6,04 a 6,55 en época de lluvia. Con respecto a los metales en las quebradas de la cuenca, para estos 4 metales ( $\mathrm{Al}, \mathrm{Fe}, \mathrm{Cu}, \mathrm{Zn}$ ) se han distribuido con la siguiente tendencia en sus concentraciones medias $\mathrm{Fe}(12,06)>\mathrm{Al}(4,47)>\mathrm{Zn}(3,03)>\mathrm{Cu}(1,69)$, esta tendencia tiene cierta similitud a lo registrado por Alonso et al. (2004) cuando analizó la presencia de metales pesados en el agua superficial en la cuenca del rio Guadiamar (Andalucía - España) que registro una tendencia de $\mathrm{Zn}>\mathrm{Cu}>\mathrm{Pb}>\mathrm{Cd}$. 


\section{Índices de Calidad del Agua}

\section{Índice de calidad ICA-PE}

Con los datos de monitoreo anual se han determinado los índices de calidad ambiental en el agua superficial tanto en ríos como en quebradas de la cuenca Chancay - Lambayeque. El índice de La calidad en los ríos ha sido siempre mayor que en las quebradas, pero que a nivel de los cuatro años del estudio han tenido una calificación en promedio de BUENA. En cuanto al menor índice de calidad determinado para los ríos y quebradas, este se ha presentado en el año 2015, mientras que para el año 2013 se obtuve el mejor índice, con el calificativo de Excelente (ver tabla 3).

Tabla 3

Índice de calidad ICA-PE de cuerpos de agua en la cuenca Chancay- Lambayeque en los años 2013 a 2016

\begin{tabular}{|c|c|c|c|c|c|}
\hline \multirow{2}{*}{ Recurso hídrico } & \multicolumn{4}{|c|}{ Año } & \multirow{2}{*}{ Promedic } \\
\hline & 2013 & 2014 & 2015 & 2016 & \\
\hline Ríos & 92,28 & 97,07 & 91,26 & 94,90 & 93,88 \\
\hline Quebradas & 87,94 & 59,50 & 51,38 & 72,23 & 67,76 \\
\hline \multirow{2}{*}{ Ríos + Quebradas } & 90,11 & 78,29 & 71,32 & 83,57 & 80,82 \\
\hline & Excelente & Buena & Regular & Buena & Buena \\
\hline
\end{tabular}

Ca tegorías: Excelente (90-100); Buena (75-89); Regular (45-75); Mala (30-45); Pésima (0-29)

\section{Índice de calidad NSF- WQI}

Aplicando la metodología de NSF - WQI a los datos de monitoreo anual del agua superficial en la cuenca Chancay - Lambayeque, se han calculado los índices de calidad ambiental tanto de ríos como de quebradas dando como resultado el de una calidad en promedio de buena. Por otro lado, el menor índice de calidad para los ríos y quebradas se ha presentado en el año 2016 y la mejor en el año 2013 (ver tabla 4).

Tabla 4

Índice de calidad NFS-WQI de cuerpos de agua en la cuenca Chancay- Lambayeque en los años 2013 a 2016

\begin{tabular}{|c|c|c|c|c|c|}
\hline \multirow{2}{*}{ Recurso hídrico } & \multicolumn{4}{|c|}{ Año } & \multirow[t]{2}{*}{ Promedio } \\
\hline & 2013 & 2014 & 2015 & 2016 & \\
\hline Ríos & 71,88 & 71,93 & 64,75 & 70,60 & 69,79 \\
\hline Quebradas & 76,91 & 76,72 & 78,92 & 63,30 & 73,96 \\
\hline \multirow{2}{*}{ Ríos + Quebradas } & 74,40 & 74,33 & 71,84 & 66,95 & 71,88 \\
\hline & Buena & Buena & Buena & Regular & Buena \\
\hline
\end{tabular}

Categorías: Excelente (91-100); Buena (71-90); Regular (51-70); Pobre (26-50); Muy pobre (0-25) 
El índice de calidad del agua determinado en este estudio mediante las dos metodologías indican que tiene la categoría de "buena", el índice ICA-PE estaba entre 71,32 a 90,11 mientras que el índice NSF-WQI entre los 66,95 a 74,40, este último índice es muy simular al obtenido en el año 2010 por Abdul Hameed et al. (2010) para el rio Tigris (Iraq), pero que a su vez es inferior al registrado por Dimnri et al. (2021) para el rio Ganga (India) que presentaba la categoría de excelente a bueno (WQI>90) o el determinado por Effendi et al. (2015) para el rio Ciambulanwung (Indonesia) con un índice NSF-WQI entre 87 y 88 , por otro lado, este índice NSF-WQI determinado para la cuenca Chancay - Lambayeque es muy superior a los obtenidos por Olguin et al. (2010) cuando determino los índices ICA (índice de Calidad del Agua adecuado del NSF.WQI) para el caso de la subcuenta del rio Sordo en Xalap, Veracruz - México) que oscilaba entre 23,54 y 63,19.

\section{CONCLUSIONES}

Las concentraciones de parámetros fisicoquímicos y microbiológicos de ríos en su mayoría no superan los valores indicados en los Estándares de Calidad Ambiental (ECA) del agua en la Categoría 3 (agua para riego de vegetales y bebida de animales), por el contrario, las quebradas presentan un $\mathrm{pH}$ fuera del rango del ECA $(6,5-8,5)$ y en lo que corresponde a los cuatro elementos contaminantes como son el aluminio, hierro, cobre y zinc estos sobrepasan el valor ECA en tres de las cuatro quebradas.

La investigación ha permitido evaluar la calidad de los cuerpos de agua de la cuenca Chancay - Lambayeque, ubicada al norte del territorio peruano, mediante dos índices de calidad y cuyos resultados son muy similares entre ambas metodologías, estos resultados ayudan a comprender que la calidad de los ríos y quebradas de la cuenca puede ser afectada por las actividades antropogénicas y procesos de meteorización que tienen lugar, especialmente en la parte alta de la cuenca.

\section{REFERENCIAS BIBLIOGRÁFICAS}

Abbasi T. and Abbasi S.A. (2012). Water Quality Indices. Elsevier publications, Amsterdam, The Netherlands.

Abdul Hameed M. Jawad Alobaidy, Bahram K. Maulood, Abass J. Kadhem. (2010). "Evaluating Raw and Treated Water Quality of Tigris River within Baghdad by Index Analysis". Journal Water Resource and Protection, 2, 629-635. doi:10.4236/jwarp.2010.27072

Alonso, E., Santos, A. Callejon, M. \& Jimenez, J.C. (2004). Speciation as a screening tool for the determination of heavy metal surface wáter pollution in the Guadiamar river basin. Chemosphere. Volume 56, Issue 6, pages 561-570. https://doi.org/10.1016/j.chemosphere.2004.04.031

ANA (2013). Informe tecnico del primer informe monitoreo participativo de calidad del agua superficial- 2013 en la Cuenca Chancay - Lambayeque. Ministerio de Agricultura. https://repositorio.ana.gob.pe

ANA (2014). Informe tecnico $\mathrm{N}^{\circ}$ 006-2014-ANA-PMGRH/ChancayLambayeque/MCL. Ministerio de Agricultura. https://repositorio.ana.gob.pe

ANA (2015). Informe tecnico $\mathrm{N}^{\circ}$ 018-2014-ANA-AAAJZ-V-SDGCRH/FZAY. Ministerio de Agricultura. https://repositorio.ana.gob.pe

ANA (2016). Informe tecnico $\mathrm{N}^{\circ}$ 021-2014-ANA-AAAJZ-V-SDGCRH/MCL. Ministerio de Agricultura. https://repositorio.ana.gob.pe 
ANA, (2018). Metodología para la determinación del índice de calidad de agua ICA-PE, aplicado a los cuerpos de agua continentales superficiales. Ministerio de Agricultura y Riego, Perú. https://hdl.handle.net/20.500.12543/2440

Bordalo, A. A. (2006). A water quality index applied an international shared river basin: The case of the Douro River, Environmental Management. 38, pp 910-920. https://doi.org/10.1007/s00267-004-0037-6

Bordalo, A. A., Nilsumranchit, W. and Chalermwat, K. (2001). "Water Quality and Uses of the Bangpakonk River (Eastern Thailand)," Water Research, Vol. 35, No. 15, pp. 3635-3642.

Brown, R. M., McClelland, N. I., Deininger, R. A. \& O'Connor, M. F. (1972). A water quality index - crashing the psychological barrier. In: Indicators of Environmental Quality. Springer, Boston, MA, pp. 173-182.

Canadian Council of Ministers of the Environment (2001). Canadian Water Quality Guidelines for the Protection of Aquatic Life: CCME Water Quality Index 1.0, User's Manual. https://www.ccme.ca/en.

Călmuc, V. A., Călmuc, M., Țopa, C. M., Timofti, M., Iticescu, C., \& Georgescu, L. P. (2018). Various methods for calculating the water quality index. Annals of the "Dunarea de Jos" University of Galati. Fascicle II, Mathematics, Physics, Theoretical Mechanics, 41(1),

171-178. https://doi.org/10.35219/ann-ugal-math-phys-mec.2018.2.09

Cude, C. (2001). "Oregon Water Quality Index: A Tool for Evaluating Water Quality Management Effectiveness," Journal of American Water Resource Association, Vol. 37, No. 1, pp. 125-137.

Dimri, D., Daverey, A., Kumar, A. \& Sharma, A (2021). Monitoring water quality of River Ganga using multivariate techniques and WQI (Water Quality Index) in Western Himalayan region of Uttarakhand, India. Environmental Nanotechnology, Monitoring \& Management. Volume 15, may 2021, 100375.

https://doi.org/10.1016/j.enmm.2020.100375

Effendi, H., Romanto, \& Wardiatno, Y. (2015). Water Quality Status of Ciambulawung River, Banten Province, Based on Pollution Index and NSF-WQI. Procedia Environmental Sciences, 24, 228-237. https://doi.org/10.1016/j.proenv.2015.03.030

El-Gafy, M. N., Faria, A., El-Bahrawy, A., Khalifa, A., El-Basiony, E. and Abdelmotaleb, M. (2005). "Decision Support System for Evaluation the Groundwater Quality," Emirates Journal for Engineering Research," Vol. 10, No. 1, pp. 69-78.

Fulazzaky, M. A. (2009). "Water Quality Evaluation System to Assess the Brantas River Water," Water Resource Management, Vol. 23, No. 14, pp. 3019-3033.

Gómez, S. E., Menni, R. C. Naya, J. G. and Ramirez, L. (2007). "The Physical-Chemical Habitat of the Buenos Aires Pejerrey, Odontesthes bonariensis (Teleostei, Atherinopsidae), with a Proposal of a Water Quality Index," Environmental Biology of Fishes, Vol. 78, No. 2, pp. 161-171.

Hallock, D. (2002). “A Water Quality Index for Ecology's Stream Monitoring Program," Technical Report, Washington Department of Ecology, Environmental Assessment Program, Olympia.

Horton, R. K. (1965). "An Index Number System for Rating Water Quality," Journal of Water Pollution Control Federation, Vol. 37, No. 3, pp. 300-306. https://doi.org/10.1016/B978-0-08-017005-3.50067-0

Liou, S. M., Lo, S. L. and Wang, S. H. (2004). "A Generalized Water Quality Index for Taiwan," Environmental Monitoring and Assessment, Vol. 96, No. 1-3, pp. 35-52. 
MINAGRI. (2016). Protocolo nacional para el monitoreo de la calidad de los recursos hídricos superficiales. In Autoridad Nacional del Agua (p. 92). http://repositorio.ana.gob.pe/handle/20.500.12543/209

MINAM. (2017). Aprueban Estandares de Calidad Ambiental (ECA) para Agua y establecen disposiciones complementarias. http://www.minam.gob.pe/wpcontent/uploads/2017/06/DS-004-2017-MINAM.pdf

Olguín, E. J., González-portela, R. E., Sánchez-galván, G., \& Zamora-, J. E. (2010). Contaminación de ríos urbanos: El caso de la subcuenca del río Sordo en Xalapa, Veracruz, México Resumen. Rev Latinoam Biotecnol Amb Algal, 1(2), 178-190. http://www.solabiaa.org/ojs3/index.php/RELBAA/article/view/23

Ott, W. R. (1978). "Environmental Indices: Theory and Practice," Ann Arbor Science Publishers Inc., Ann Arbor, Michigan, USA.

Pandey, M. and Sundaram, S. M. (2002). Trend of water quality of river Ganga at Varanasi using WQI approach, International Journal of Ecology and Environmental Science. New Delhi: International Scientific Publication. 22, pp 139-142.

Pesce, S. F. and Wunderlin, D. A. (2000). "Use of Water Quality Indices to Verify the Impact of Córdoba City (Argentina) on Suquìa River," Water Research, Vol. 34, No. 11, pp. 29152926.

Ram, R. S. and Anandh, H. (1996). Water quality index of some Indian rivers, Indian Journal of Environmental Health, NEERI Nagpur. 38 (1), pp 21-34.

Rodríguez Flores, R. G. (2019). Evaluación de la calidad del agua en la cuenca ChancayLambayeque (Perú) en términos de índices de calidad del agua Ica-Pe y NSF-WQI. Universidad Nacional del Callao, Perú. http://repositorio.unac.edu.pe/handle/UNAC/5578

Sargaonkar, A. and Deshpande, V. (2003). "Development of an Overall Index of Pollution for Surface Water Based on a General Classification Scheme in Indian Context," Environmental Monitoring and Assessment, Vol. 89, No. 1, pp. 43-67. https://doi.org/10.1023/A:1025886025137

Sinha, D. K. and Saxena, A. (2006). "Statistical Assessment of Underground Drinking Water Contamination and Effect of Monsoon at Hasanpur, J.P.Nagar (Uttar Pradesh, India)," Journal of Environmental Science and Engineering, Vol. 48, No. 3, pp. 157-164.

Smith, D. G. (1990). A better water quality indexing system for rivers and streams. Water Research 24 (10), 1237-1244.

Štambuk-Giljanović, N. (2003). "Comparison of Dalmatian Water Evaluation Indices," Water Environment Research, Vol. 75, No. 5, pp. 388-405. 